\title{
Como usar a abordagem da Política Informada por Evidência na saúde pública?
}

How to use the Evidence-informed Policy approach in public health?

Maíra Catharina Ramos ${ }^{\mathbf{1}}$ Everton Nunes da Silva ${ }^{2}$

RESUMO A Política Informada por Evidência (PIE) surgiu para identificar as melhores estratégias disponíveis, nos aproximando do processo de tomada de decisão em políticas públicas. Contudo, para que ocorra, depende do acesso ao conhecimento, que deverá ser utilizado de forma transparente e sistemática. Assim, tem-se por objetivo apresentar como valer-se da PIE, mostrando os passos necessários para utilizá-la no processo de tomada de decisão em saúde pública. Foi realizada revisão de literatura em duas bases, sendo selecionados nove estudos, após leitura completa do texto. $\mathrm{O}$ artigo foi dividido em três grandes blocos, cada um contendo as principais perguntas a serem respondidas durante a elaboração de uma síntese de evidência.

PALAVRAS-CHAVE Pesquisa médica translacional. Política Informada por Evidências. Sistemas de saúde.

ABSTRACT The Evidence-informed Policy (EIP) emerged to identify the best strategies available, bringing us closer to the decision-making process in public policy. However, for this to occur, it depends on access to knowledge, which should be used in a transparent and systematic way. Thus, the goal is to present how to use the EIP, showing the steps necessary to use it in the decisionmaking process in public health. Literature review was carried out in two databases, being selected nine studies, after full text reading. The article was divided into three large blocks, each containing the main questions to be answered during the elaboration of a synthesis of evidence.

KEYWORDS Translational medical research. Evidence-informed Policy. Health systems.

1 Universidade de Brasília (UnB) - Brasília (DF),

Brasil.

mairacramos@gmail.com

2 Universidade de Brasília (UnB) - Brasília (DF),

Brasil.

evertonsilva@unb.br 


\section{Introdução}

O uso do conhecimento científico para a tomada de decisão é uma prática comum às ciências, como ocorre no campo biomédico e jurídico, com o uso da medicina baseada em evidência e do direito baseado em evidência, respectivamente. Não obstante, o uso do conhecimento científico para informar o processo de formulação e implementação de uma política começou a ser utilizado na década de 1970, com a então 'política baseada em evidência' ${ }^{\prime, 2}$.

Entretanto, há um grande abismo entre o que se tem de evidência científica e o que se é utilizado na prática; ou, em uma linguagem mais pragmática, entre o 'saber e o fazer', o que denomina-se know-do gap. Uma das formas de superar esse abismo envolve a tradução do conhecimento, do inglês knowledge translation, que é um processo interativo que inclui síntese, disseminação, intercâmbio e aplicação ética do conhecimento, sendo a ponte entre o pesquisador e o tomador de decisão'.

Nesse contexto, para o aumento da efetividade das políticas públicas, por meio de evidências científicas, é necessária a inserção de dois mecanismos importantes: o político e o organizacional. O processo da política é inseparável dos valores políticos, da persuasão e da negociação. No contexto da política, algumas evidências possuem maior relevância que posicionamentos políticos, como a efetividade de medicamentos para uma doença de grande valor social, a fixação e a alocação de profissionais qualificados em áreas remotas e vulneráveis, a economia social, entre outros.

De forma geral, os tomadores de decisão articulam suas próprias experiências com valores e interesses sociais, influenciados, também, pela disponibilidade de recursos e pela oportunidade política. Por isso, a dinâmica política pode ser moldada pela forma como líderes políticos pretendem segui-la, bem como pela influência de legisladores, lobistas, stakeholders, mídia, opinião pública, entre outros.

Entretanto, o sucesso das ações políticas depende mais da efetividade das intervenções do que da vontade do tomador de decisão, propriamente ${ }^{\mathbf{1}, \mathbf{2}}$. Intervenções sem efeito, ainda que realizadas com muita vontade política, tendem a ser extintas ou reformuladas em sua totalidade. Recentemente, o Brasil modificou o esquema vacinal para Papiloma Vírus Humano (HPV). Apesar dos esforços do Ministério da Saúde, a mudança do cenário de aplicação da vacina, passando das escolas para Unidades Básicas de Saúde, causou um efeito negativo, reduzindo a cobertura da vacina de HPV ${ }^{3}$.

A 'política baseada em evidência' soaria ambiciosa demais para o contexto político, inerente aos conflitos, trade-offs e aos compromissos políticos. Segundo ele, foi por esse motivo que alguns autores defenderam o uso mais modesto do termo Política Informada por Evidência (PIE), cujo objetivo não é o de que os tomadores de decisão baseiem suas políticas em outras que obtiveram sucesso, pois os contextos e a janela de oportunidade local certamente serão diferentes. O propósito, contudo, é informar os gestores sobre as estratégias disponíveis, bem como indicar as melhores evidências da literatura. $\mathrm{O}$ gestor deverá escolher as estratégias que melhor se adequam à sua janela de oportunidade, modificando-as sempre conforme o seu contexto. Nesse sentido, as políticas dificilmente serão iguais umas às outras ${ }^{2}$.

Assim, a PIE nos aproxima do processo de tomada de decisão em políticas públicas informadas pelas melhores evidências disponíveis. Contudo, para que isso ocorra, ela depende do acesso ao conhecimento disponível, que deverá ser utilizado de forma transparente, sistemática e criteriosa. Saber utilizar a PIE é essencial para que se tenha bons resultados no processo de tomada de decisão, em especial, na saúde, onde se observam inúmeros fatores que influenciam diretamente esse processo.

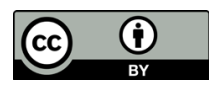

Este é um artigo publicado em acesso aberto (Open Access) sob a licença Creative Commons Attribution, que permite uso, distribuição e reprodução em qualquer meio, sem restrições, desde que o trabalho original seja corretamente citado. 
$\mathrm{Na}$ área da saúde, existe um crescente interesse em todo o mundo em garantir a elaboração de políticas públicas informadas por evidência 4 . Elaborar uma política pública de saúde baseada em evidência permite a melhoria do desempenho do sistema de saúde, além de evitar as iniquidades provenientes de políticas públicas mal formuladas. Para que isso aconteça, é preciso envolver, além dos pesquisadores, os formuladores de políticas e os stakeholders, a fim de promover maior compreensão da política de saúde. Afinal, são esses que elaboram e implementam políticas públicas.

Diante o exposto, este artigo tem por objetivo apresentar como se utilizar da PIE na saúde pública.

\section{Métodos}

Foi realizada revisão de literatura com a busca ('evidence informed policy making'[Title/Abstract]) OR ('evidence informed policymaking'[Title/Abstract])
OR ('evidence informed policy'[Title/ Abstract]) OR ('Evidence-Informed Policymaking'[Title/Abstract]) AND ('public health'[Title/Abstract]] nas bases Pubmed e Biblioteca Virtual em Saúde (BVS). A busca resultou em 25 estudos.

Após a remoção das duplicatas, restaram 24 estudos únicos, dos quais foram selecionados 14, após a leitura dos resumos. Destes, foram selecionados 9, após a leitura completa do texto. Incluíram-se apenas os estudos que abordaram o uso da PIE na saúde, excluindo-se os estudos sem publicação completa ou que não abordavam a PIE.

Ao fim, sistematizaram-se os estudos de forma a oferecer um passo a passo de como utilizar a PIE na saúde pública. Para tal, os estudos foram divididos em blocos, que apresentam os procedimentos necessários para usar a PIE no processo de tomada de decisão em saúde pública. A figura 1 é a representação gráfica de cada um desses blocos, evidenciando as perguntas que devem ser respondidas em cada seção. 
Figura 1. Passo a passo para o uso da Política Informada por Evidência na saúde pública

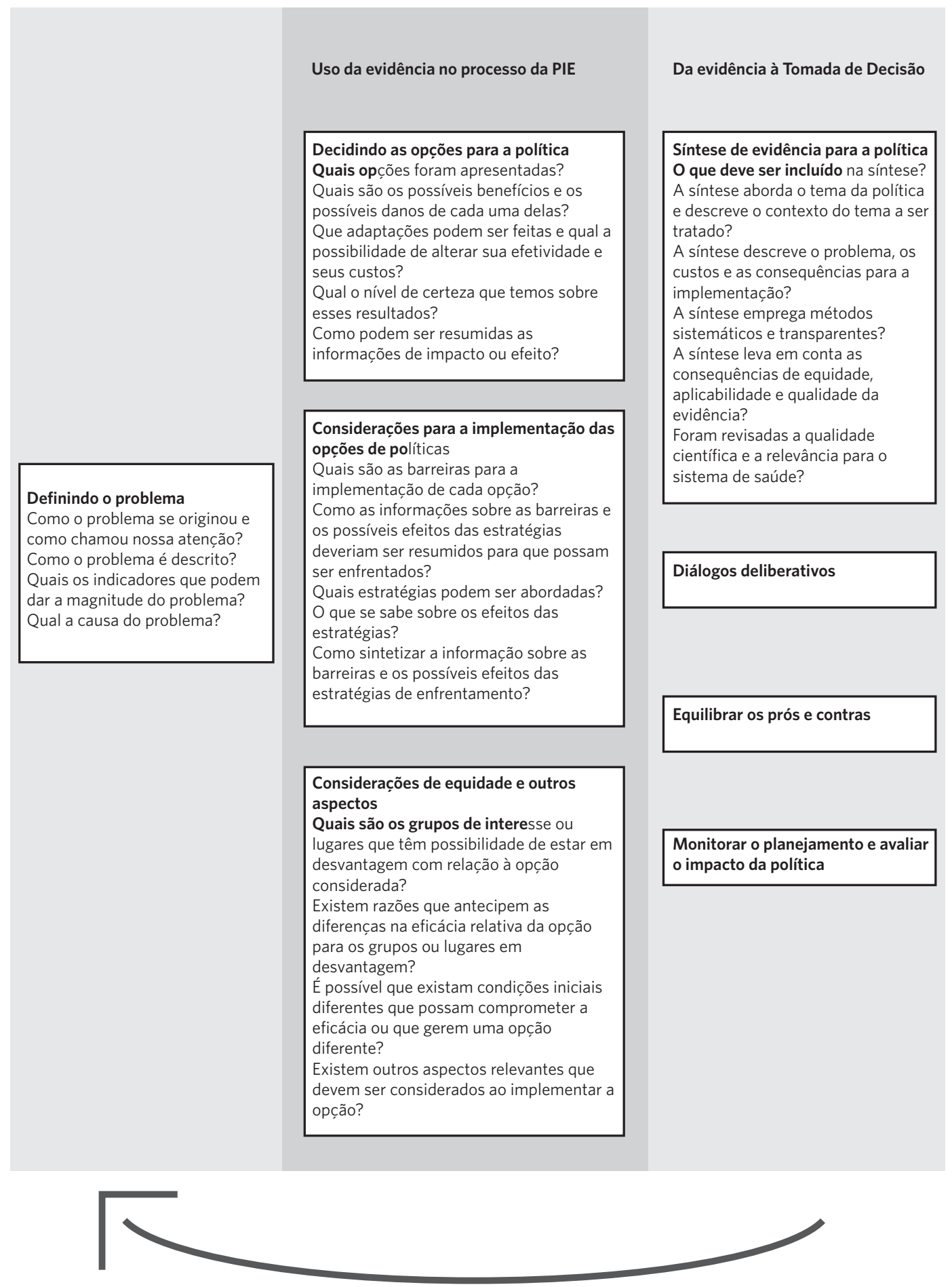




\section{Definindo o problema}

O primeiro bloco da PIE visa a descrever o problema de forma consistente, identificando a sua origem e os principais pontos que chamam a atenção para ele, sua magnitude e sua causa ${ }^{5}$.

O primeiro procedimento desse bloco é pensar em como o problema é descrito e identificar as opções para resolvê-lo. Diferentes atores descrevem o mesmo problema de forma distinta, gerando, assim, opções distintas para a implementação de uma estratégia. Por esse motivo, a definição do problema, apesar de parecer fácil, é uma tarefa que requer atenção: não pode ser rápida ou superficial. Em caso de má definição, os passos seguintes podem ser comprometidos.

Na definição do problema, também é necessário mensurá-lo mediante indicadores selecionados em diferentes fontes, sendo esse o segundo procedimento do bloco. Se o problema for descrito em termos de fatores de risco e carga de doença, os dados epidemiológicos são fontes de informação essenciais para estimar sua grandeza. Entretanto, se os indicadores utilizados dizem sobre o uso dos serviços de saúde, o ideal são estudos de acesso, qualidade dos serviços, gastos e até mesmo de iniquidades geradas. Há, ainda, a possibilidade de o problema ser descrito nos termos de mecanismos e estratégias de implementação. Incluem como fonte documentos governamentais, estudos coletados pelo governo ou estudos que descrevam e analisem os mecanismos ou as estratégias de implementação. Nesses casos, as fontes de informação são altamente variáveis e podem ser difíceis de ser localizadas ${ }^{5}$. Ainda quanto à magnitude, pode-se utilizar de comparadores para determinar o quão grande é o problema. Comparar o mesmo problema em situações distintas - entre regiões, países ou em séries de tempo - pode nos mostrar o tamanho do problema.
Por fim, para o bloco 'Descrevendo o problema', é preciso conhecer a causa do problema, sendo esse o terceiro procedimento. Entendê-la ajuda a identificar opções apropriadas e delimitá-las melhor. No campo da saúde pública, as causas dos problemas são incertas e complexas, e, por isso, na maioria das vezes, não se trata de um processo linear e evidente. Assim, existem algumas estratégias que podem ajudar na definição das causas dos problemas, como a brainstorm, a revisão de literatura científica ou o uso de marcos de referência.

\section{Usando a evidência para o processo da PIE}

As próximas três etapas necessitam de uma fonte de informação para que sejam esclarecidas. As evidências estão relacionadas a fatos utilizados para apoiar conclusões, e, por isso, não se trata de ter uma conclusão ${ }^{6}$. Nota-se que nem toda evidência possui o mesmo grau de confiabilidade, sendo, por isso, necessária a avaliação do desenho de estudo, dos possíveis vieses, de sua precisão e da força de associação que é reportada. Assim, é recomendável que se utilize a pirâmide da evidência científica no processo da PIE, onde os overviews, as revisões sistemáticas e as metanálises são as melhores evidências disponíveis.

\section{Decidindo as opções para a política}

A segunda etapa desse passo consiste na decisão sobre as opções encontradas para a política. Devem ser consideradas questões como: quais opções foram apresentadas? $\mathrm{O}$ que se sabe sobre seus impactos ou efeitos? Qual o nível de certeza que se tem sobre esses resultados? Como posso adaptá-las ao meu problema5?

A partir das propostas apresentadas, devem-se justificar, de forma explícita, os motivos que levaram à escolha ou à exclusão 
das opções apresentadas - lembrando que a escolha de uma opção para a política não exclui, necessariamente, outra. Pode haver opções distintas para alcançar um resultado melhor, desde que observados os possíveis efeitos e impactos que tal mudança poderá ocasionar a diferentes grupos sociais.

Para selecionar as opções que melhor se adequam ao seu problema, é preciso considerar mecanismos alternativos de prestação, financiamento e governança que abordem o problema relatado; marcos teóricos desenvolvidos para um problema específico; intervenções descritas em revisões sistemáticas ou overviews (metarrevisões); e a forma com que o problema tem sido abordado em outras localidades. Caso necessário, ainda podem-se realizar consultas com informantes-chave ${ }^{7}$.

\section{Considerações para a implementação das opções de políticas}

Implementar ações é uma tarefa difícil, pois requer mudança em vários níveis, como a mudança de comportamento de atores-chave e mudanças organizativas ${ }^{5}$. Quanto melhor relatadas as possíveis barreiras para a sua implementação, maiores as chances de sucesso. Para tal, necessita-se de uma investigação estruturada na literatura para a identificação de barreiras, para que nenhuma seja subestimada.

Considera-se a existência de diferentes níveis de barreiras, podendo ser ligadas: aos usuários ou profissionais do sistema de saúde (motivação para mudanças, conhecimentos, atitudes, competências, acesso ao cuidado etc.); a limitações do sistema de saúde (escassez de recursos, processos inadequados, falta de governança, excesso de burocracia etc.); ou, ainda, a limitações sociais e políticas (práticas sociais, janela de oportunidade política, conflitos de interesse etc.).

Da mesma forma, é necessário conhecer os efeitos das estratégias de implementação, localizando e avaliando as evidências disponíveis. Basear-se em evidências possibilita a avaliação dos efeitos de cada estratégia de implementação considerada, bem como a confiança que pode ser colocada nessas estimativas.

Por fim, é necessário sintetizar essas informações. É preciso deixar claros a evidência e os critérios utilizados para caracterizar essas barreiras; informar as vantagens e desvantagens das estratégias de implementação, a qualidade da evidência e quaisquer limitações identificadas.

Muitas vezes, as evidências sobre os efeitos das estratégias são limitadas, porém, se realizadas de forma superficial, podem acarretar riscos que comprometerão sua implementação. Quanto mais sistemático e transparente esse processo, maiores as chances de sucesso na implementação de uma ação ${ }^{8}$.

\section{Considerações de equidade e outros aspectos}

Conhecer a eficiência de uma política ou de um programa não é suficiente no processo de tomada de decisão. Faz-se necessário considerar, também, o seu impacto sobre as desigualdades sociais. Ademais, é preciso pensar se há a possibilidade de algum grupo estar em desvantagem com relação à opção considerada; se existe eficácia relativa a grupos distintos; se condicionantes sociais podem comprometer a eficácia de determinada estratégia; ou, ainda, se há outros aspectos relevantes que podem interferir na implementação da ação 9 .

Uma das formas de identificar os grupos sociais que merecem atenção, a fim de aumentar as iniquidades com a implementação de uma ação, é o PROGRESS, acrônimo formado com as primeiras letras (em inglês) das sete formas que podem ser usadas para descrever um grupo ${ }^{5}$. São elas: i) local de residência; ii) raça/etnia/cultura; iii) ocupação; iv) gênero; v) religião; vi) educação; e vii) situação socioeconômica. 


\section{Da evidência à tomada de decisão}

Uma vez que se tenha concluído a etapa do uso da evidência para o processo da PIE, é preciso utilizá-la para a tomada de decisão. Nessa seção, existem quatro procedimentos: i) síntese de evidência para a política; ii) diálogos deliberativos; iii) equilibrar os prós e os contras; e iv) monitorar o planejamento e avaliar os efeitos da política.

\section{Síntese da evidência para a política}

Uma síntese para políticas representa um conjunto de evidências cujo ponto de partida é um tema de saúde, não propriamente a evidência que tenha sido produzida ou identificada. Primeiramente, deve-se focar em revisões sistemáticas ou em overviews disponíveis na literatura, somente partindo para a sua elaboração em último caso.

A primeira pergunta a ser respondida é: 'o que deve ser incluído na síntese?'. Segundo a ferramenta SUPPORT ${ }^{5}$, é preciso incluir as mensagens-chave; o resumo executivo com a sinopse do problema, opções de política e de implementação; e o relatório completo, que é dividido em três seções. A primeira delas, o problema, deve conter como isso chegou a ser um problema, como ele pode ser descrito, qual o seu tamanho e os fatores associados a esse problema.

A segunda seção, opções de política, deve apresentar os impactos prováveis, as considerações de equidade, custos e custo-efetividade, além da necessidade de monitoramento e avaliação. A terceira seção, considerações de implementação, deve apresentar as barreiras e as estratégias de implementação das opções. Observem que todos os itens necessários já foram levantados em algum momento anteriormente. Dessa forma, a síntese da evidência nada mais é do que a sistematização clara e transparente dos achados na literatura.

Ainda que as sínteses incluam o descrito acima, elas precisam responder às perguntas a seguir: i) a síntese aborda o tema da política e descreve seu contexto?; ii) a síntese descreve o problema, os custos e as consequências para a implementação?; iii) a síntese emprega métodos sistemáticos e transparentes?; iv) a síntese leva em conta as consequências de equidade, aplicabilidade e qualidade da evidência?; v) foram revisadas a qualidade científica e a relevância para o sistema de saúde?

Essas questões dizem respeito à qualidade da síntese de evidência. Quanto maior o número de questões cujas respostas sejam 'sim' para os itens acima, menor o risco de a síntese possuir algum viés que possa vir a interferir em seu resultado.

\section{Diálogos deliberativos}

Depois de concluídas, as sínteses de evidência poderão ser utilizadas de diversas formas: divulgação da síntese, informação adaptada, meios de comunicação massivos, apresentações presenciais, entrevistas, grupos focais, pesquisas, audiências públicas, entre tantas outras maneiras.

O que muda de um meio para outro, entretanto, é a capacidade de envolver esses grupos de forma a dar voz a eles, havendo, assim, um processo comunicativo - e não apenas informativo - da PIE. A esse processo, deu-se o nome de diálogos deliberativos.

Os diálogos deliberativos são direcionados para atingir grupos específicos, que incluem usuários e o público, em que funcionários eleitos se reúnem com essas partes para promover o processo de consenso. Nesse consenso, busca-se agregar valor à síntese de evidência, ajudando a esclarecer os problemas e as soluções, desenvolvendo um entendimento comum, contribuindo, assim, para o desenvolvimento e a implementação de políticas efetivas e da governança e da democracia 5 .

\section{Equilibrar prós e contras}

Toda tomada de decisão deve considerar o 
julgamento dos prós e contras. Deve-se ter conhecimento dos resultados desejados e dos indesejáveis, ainda que sejam difíceis de serem previstos. Equilibrar os prós e os contras prováveis da política proposta permite considerar informações importantes, evitando, assim, a negligência de possíveis efeitos negativos. Ademais, permite também entender a razão por trás das políticas de saúde ${ }^{\mathbf{5}}$.

Tal procedimento é de relevância por evitar maiores iniquidades em saúde. Se implementada política de saúde que produz mais efeitos negativos, seja no âmbito social ou econômico, é preciso se repensar se a estratégia escolhida foi a melhor possível. Por tal motivo, o uso da literatura para ajudar nesse procedimento, ainda que com julgamentos dos formuladores, mostra-se necessário por produzir evidências robustas e transparentes.

Para identificar os pontos positivos e negativos da evidência, a ferramenta SUPPORT ${ }^{5}$ indica cinco perguntas usadas para avaliar os prós e contras das opções das políticas. A primeira delas é 'que opções estão sendo comparadas?'. Comparam-se os diferentes cenários para cada uma das opções, a considerada e a comparativa. Normalmente, essa comparação é um status quo, que pode ser fundamental - onde as características das opções devem ser as mesmas, sendo excluídas, caso contrário; importante, porém não fundamental - assim como a anterior, é excluída em casos de divergência entre as opções, porém, com menor certeza de que os resultados seriam iguais no cenário escolhido; ou irrelevante - quando existe maior certeza de que os resultados serão os mesmos apesar dos cenários.

A segunda pergunta é: 'quais são os resultados potenciais mais importantes das opções sendo comparadas?'. Na área da saúde, os resultados podem ser de aumento do acesso aos serviços de saúde, redução de iniquidades, redução de custos, melhor gestão de serviços, entre outros. Identificar os resultados de maior potencial ajuda a garantir que as consequências mais importantes não sejam negligenciadas ou subestimadas. A terceira pergunta, 'qual é a melhor estimativa de impacto das opções sendo comparadas para cada resultado importante?', é o balanceamento entre o que se ganha e o que se perde ao implementar uma ação em detrimento da outra. É o princípio do trade-off. Além disso, é preciso saber diferenciar os efeitos de ordem relativa dos absolutos.

Para responder à quarta pergunta, 'qual o grau de confiabilidade dos formuladores de políticas e de outros nos impactos estimados?', é preciso ter conhecimento sobre a confiabilidade dos formuladores e dos resultados esperados. Se os estudos utilizados tiverem limitações metodológicas ou vieses conhecidos, os efeitos não serão confiáveis. Por isso é tão importe que uma avaliação de qualidade metodológica dos estudos primários seja bem realizada. A avaliação e a qualidade metodológica ajudam a conhecer os vieses dos estudos, bem como os pontos mais críticos na metodologia e no resultado.

Por fim, faz-se a pergunta: 'é um modelo econômico formal suscetível de facilitar processos de tomada de decisões?'. Para tal, é preciso realizar modelos econômicos que indiquem a sustentabilidade da opção a ser considerada. Ademais, um modelo econômico também indica possíveis variações nos custos a partir de uma análise de sensibilidade, indicando uma porcentagem que pode extrapolar os recursos previstos. Assim, o tomador de decisão poderá avaliar se, ainda que no pior cenário, a opção considerada ainda é mais vantajosa que a de comparação.

\section{Monitorar o planejamento e avaliar o impacto da política}

O último procedimento desse passo é monitorar o planejamento e avaliar o impacto da política. Frequentemente, o termo monitorar é utilizado como forma de descrever o processo sistemático de coleta de dados que irá 
informar os tomadores de decisão, enquanto avaliar sugere menção aos resultados. Para avaliar e monitorar o planejamento, fazem-se quatro perguntas: i) é necessário o monitoramento?; ii) o que deve ser medido?; iii) a avaliação do impacto deve ser feita?; iv) como a avaliação do impacto deve ser feita?

Em alguns casos, o monitoramento da política não é necessário, ou por já existir um sistema de monitoramento semelhante implementado, ou por possuir custos muito elevados, ou por não existirem indicadores que trarão a dimensão correta do dado. $\mathrm{O}$ monitoramento não é vantajoso se o dado não é utilizado 5 .

A avaliação de impacto pode ser entendida como uma maneira de medir quanto dos resultados observados podem ser atribuídos a uma determinada política. Por isso, deve-se escolher a hora certa de realizar uma avaliação de impacto, ou corre-se o risco de comprometer toda uma política. Por exemplo, se for realizada a avaliação de impacto em uma política e for verificado impacto negativo, há o risco de interrupção da mesma? Se a resposta for sim, existe um comprometimento da política. Por esse motivo, indica-se a avaliação de resultado quando a intervenção está sendo implementada, onde ainda são possíveis melhorias e mudanças. Caso contrário, a avaliação de impacto pode ser limitada.

\section{Exemplos de uso da PIE na prática}

O uso da PIE na tomada de decisão tem contribuído para reduzir o know-do gap. $\mathrm{O}$ Brasil vem desenvolvendo diversas atividades para estimular o uso do conhecimento científico na tomada de decisão. Em 2007, criou-se a Rede de Políticas Informadas por Evidências (EVIPNet) no Brasil, apoiada pela Organização Mundial da Saúde (OMS) e pela Organização Pan-americana de Saúde (Opas), além de receber financiamento do Ministério da Saúde (MS).
A EVIPNet desenvolve parcerias para o desenvolvimento da própria rede e para a produção de síntese de evidências para a política, além de oferecer capacitação profissional10. A EVIPNet Brasil é uma rede promotora de troca de informações e experiências entre países e entre tomadores de decisão clínica e gerencial1', produzindo, até o momento, 13 sínteses de evidência.

Destaca-se que a maioria das sínteses disponíveis trata de doenças crônicas não transmissíveis, seja para a promoção e a prevenção da doença, seja para o tratamento. Em ambos os casos, o intuito é reduzir as taxas de prevalência dessas doenças. Há, ainda, uma síntese específica sobre adesão ao tratamento medicamentoso para pacientes portadores de doenças crônicas. A síntese em questão aborda quatro opções diferentes para enfrentar o problema: i) técnicas educacionais, motivacionais, cognitivas e desenvolvimento de sistemas de lembretes; ii) oferta de incentivos ao paciente (normalmente monetários); iii) intervenções para auxílio na gestão dos medicamentos pelo paciente; e iv) combinação de intervenções voltadas ao paciente. Todas as intervenções possuíam, em algum nível, efeitos positivos para a adesão ao tratamento de doenças crônicas, entretanto, os autores escolheram não recomendar uma opção específica.

A primeira experiência de um núcleo de práticas de PIE no Brasil foi em Piripiri, Piauí, implementado em 2010 como forma de promover o uso local do conhecimento científico na tomada de decisão e na formulação e na implementação das políticas públicas. O exemplo do Núcleo de Evidência (Nev) de Piripiri foi importante para mostrar como aproximar o uso da evidência científica em âmbito municipal, trazendo a elaboração e a implementação de políticas de saúde informadas em evidência próximas ao processo de gestão'.

Outros países do mundo também procuram na PIE assegurar que a tomada de decisão seja informada pela melhor 
evidência disponível. Assim como o Brasil, a Austrália enfrenta problemas de grandes áreas remotas e vulneráveis nos vazios assistenciais. Este país buscou na PIE estratégias de implementação de práticas para dor lombar persistente, onde o manejo poderia ser realizado por prestadores de cuidados que vivem em áreas rurais. Após a elaboração da síntese de evidência e a implementação da estratégia, a Austrália realizou um estudo de coorte prospectivo, controlado e randomizado, com duração de 12 meses para monitorar e avaliar a implementação do projeto, conforme preconizado pela PIE"1.

Como resultado, o estudo demonstrou que foi possível implementar uma intervenção educacional informada em evidência aos prestadores de cuidado que vivem em áreas remotas e vulneráveis. Os resultados demonstraram, também, eficácia no tratamento de dor lombar persistente, além de os provedores de cuidado relatarem buscar mais práticas informadas em evidências e mais comportamentos clínicos consistentes com diretrizes clínicas"1.

Algumas vezes, o uso da PIE vai além da busca e da implementação de políticas. Quando a EVIPNet começou a operacionalizar na África, o primeiro desafio foi promover a 'inspiração' de um desenvolvimento mais local - apesar do desenvolvimento econômico e social que o continente vem adquirindo nos últimos anos, ainda convive com uma desigualdade social alarmante, além de altos índices de miséria. Por isso, o uso da evidência científica combinada à evidência local é importante nesses casos.

Uma das primeiras sínteses produzidas pelo grupo foi sobre a malária, doença que acomete uma parcela preocupante da população do continente. Entretanto, o principal produto não foi a síntese em si, mas a disseminação do diálogo de políticas como forma de apoiar sistemas de saúde informados em evidência, especialmente em países de baixa e média renda, além de ajudar a construir um modelo específico de tradução do conhecimento combinado com infraestrutura, atividade e resultado, dados os contextos tão específicos, a fim de alcançar melhores resultados e impactos $^{12}$.

\section{Conclusões}

A PIE pode ser utilizada na saúde, como forma de garantir que o processo de tomada de decisão seja fundamentado na melhor evidência científica disponível, de forma sistemática e transparente. O processo da PIE foi esquematizado de forma a conter três grandes blocos, entretanto, o ciclo da política não termina após a sua implementação, sendo necessário retomar o primeiro ponto indicado: definindo o problema. 


\section{Referências}

1. Barreto JOM, Souza NM. Making progress in the use of health policies and practices informed by evidence: the Piripiri-Piaui experience. Ciênc Saúde Colet. 2013; 18(1):25-34.

2. Head BW. Toward More Evidence-Informed Policy Making? Public Adm Rev. 2016; 76(3):472-84.

3. França SB, Silva RAR, Cardoso JS, et al. Adesão das adolescentes à campanha de vacinação contra o papiloma vírus humano: no Brasil, Minas Gerais e microregião da Serra Geral. Unimontes Científica. 2017; 19(1):2-12.

4. Langlois EV, Montekio VB, Young T, et al. Enhancing evidence informed policymaking in complex health systems: lessons from multi-site collaborative approaches. Heal Res Policy Syst. 2016; 14(1):20.

5. Oxman AD, Lavis JN, Lewin S, et al. SUPPORT tools for evidence-informed health policymaking (STP). Heal Res Policy Syst. 2009 Dec 16; 7(sup.1):S1-S13.

6. Bowen S, Zwi AB. Pathways to evidence-informed policy and practice: A framework for action. PLoS Med. 2005; 2(7):600-605.

7. Shlonsky A, Mildon R. Methodological pluralism in the age of evidence-informed practice and policy. Scand J Public Health. 2014; 42:18-27.
8. Bosch-Capblanch $\mathrm{X}$, Lavis $\mathrm{JN}$, Lewin $\mathrm{S}$, et al. Guidance for Evidence-Informed Policies about Health Systems: Rationale for and Challenges of Guidance Development. Plos Med. 2012 Mar 6; 9(3):1-5.

9. Lavis JN, Røttingen JA, Bosch-Capblanch X, et al. Guidance for evidence-informed policies about health systems: Linking guidance development to policy development. PLoS Med. 2012 Mar 13; 9(3):1-6.

10. Silva SF, Souza NM, Barreto JOM. Fronteiras da autonomia da gestão local de saúde: inovação, criatividade e tomada de decisão informada por evidências. Ciênc Saúde Colet. 2014; 19(11):4427-4438.

11. Slater H, Briggs AM, Smith AJ, et al. Implementing Evidence-Informed Policy into Practice for Health Care Professionals Managing People with Low Back Pain in Australian Rural Settings: A Preliminary Prospective Single-Cohort Study. Pain Med. 2014; 15(10):1657-1668.

12. Lavis JN, Panisset U. EVIPNet Africa's first series of policy briefs to support evidence-informed policymaking. Int J Technol Assess Health Care. 2010; 26(2):229-232.

Recebido em 08/07/2017

Aprovado em 08/02/2018

Conflito de interesses: inexistente

Financiamento: não houve 